\title{
VERSITA
}

Journal of the Applied Mathematics, Statistics and Informatics (JAMSI), 8 (2012), No. 2

\section{Skeleton-based 3D Surface Parameterization Applied on Texture Mapping}

\author{
MARTIN MADARAS*, AND ROMAN ĎURIKOVIČ \\ Comenius University Bratislava
}

\begin{abstract}
Assume a 2D manifold surface topologically equivalent to a sphere with handles we propose a novel 3D surface parametrization along the surface skeleton. First, we use a global mapping of the surface vertices onto a computed skeleton. Second, we use local mapping of the surrounding area of each skeleton segment into a small rectangle whose size is derived based on the surface properties around the segment. Each rectangle can be textured by assigning the local $u, v$ texture coordinates. Furthermore, these rectangles are packed into a large squared texture called skeleton texture map (STM) by approximately solving a palette loading problem.
\end{abstract}

Our technique enables the mapping of a texture onto the surface without necessity to store texture coordinates together with the model data. In other words it is enough to store the geometry data with STM and the coordinates are calculated on the fly.

Mathematics Subject Classification 2000: 14C05, 65D17, 68U05

Additional Key Words and Phrases: skeleton, texture, mapping, parameterizations

\section{INTRODUCTION AND RELATED WORK}

Texture mapping is a commonly used and the most successful technique of improving visual quality of 3D surfaces in computer graphics. The problem of texture mapping can be formulated as retrieving a pair of texture coordinates for each surface point in order to put the surface into one-to-one correspondence with an image.

Our motivation behind this approach is to parameterize a model using a piece-wise easily parameterizable guiding structure that is dependant only on the topology of the model, not the surface. Moreover, such a parameterization would be used for generating of texture and geometry patterns around the skeleton. The textures encoding these patterns can be generated independently to the geometry and later applied on a set of models with the same skeleton branching. In a case, when the texture encodes a geometry, it can be used for procedurally generating of geometry around given skeleton tree. In addition, the param-

* e-mail: e-mail: madaras@sccg.sk

$\dagger$ e-mail: durikovic@fmph.uniba.sk

(C)ACM, 2012. This is a revision of the work published in Proceedings of the Spring Conference on Computer Graphics - SCCG2012, ISBN: 978-80-223-3211-8, Smolenice, Slovakia

DOI 10.2478/v10294-012-0010-6

CUniversity of SS. Cyril and Methodius in Trnava 


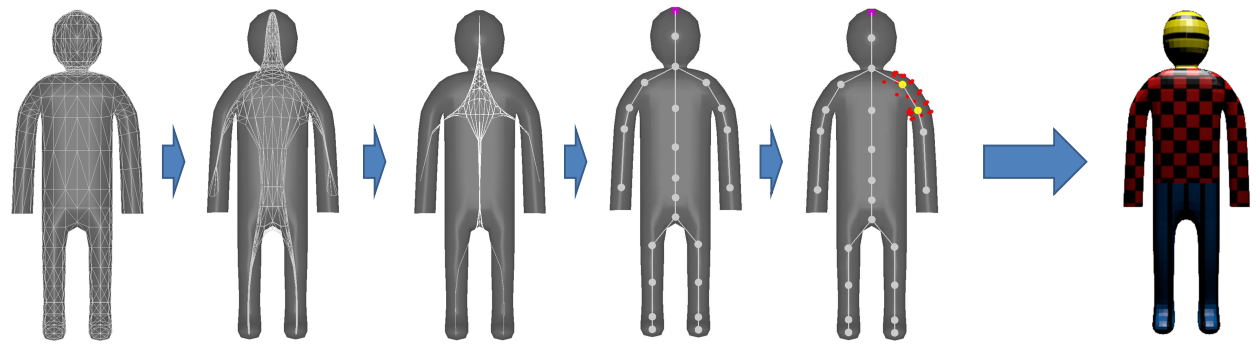

Fig. 1. (From left to right) Our method takes an input model and computes a skeleton using an iterative Laplacian smoothing. Then, using an association between each skeleton segment and vertices around it the method maps each skeleton segment into rectangular textures. Finally, the method computes a global surface to texture mapping by packing the rectangular textures into a final texture.

eterization would enable transferring of patterns between models with the same skeleton branching.

In our approach the mapping between the surface and the skeleton works in a deterministic way and the benefit of our approach is that $u v$ coordinates can be computed during rendering process. In the first stage, a skeleton is extracted from an input mesh. For skeleton extraction, the algorithm from [Au et al. 2008] with minor modifications is used. The algorithm extracts the skeleton from a closed 2D-manifold mesh using iterative Laplacian contraction. If the input is a surface with boundaries, polygon soup or point cloud, we can use the adaptation of this algorithm [Cao et al. 2010] for handling such surfaces. During the skeleton construction we store the mapping of mesh vertices collapsed into each skeleton node. Furthermore, we compute and store some surface properties of the collapsed vertices into the corresponding skeleton node. These properties serve for priority weight estimation. These weights determine the importance of segment in a sense of level of detail and control how much of space the segment requires in the final texture. With the knowledge of relative size ratio between the segment textures, we solve the packing problem and allocate an area in the final texture for each segment. In the next step, we apply segment mapping and cover allocated area for each skeleton segment. To avoid aliasing and waxy look of textures a filtering has to be applied in the texture mapping. To guarantee that each texel has a well defined local neighborhood, each rectangular texture is enclosed in horizontal direction by mirrored parts of itself and in the vertical direction by texture parts of the neighbouring segments. A graph demonstrating a workflow of our algorithm can be seen in Figure 1. The proposed technique is also suitable for surfaces with topology non-homotopic to a sphere, higher order genus and very complex branching structure, where common mapping techniques often based on unwrapping mesh into $2 \mathrm{D}$ domain may fail (see Figure 2). The reason that our method handles this problem is that the texture is remapped into several textures, thus giving each segment it's own texture space.

\section{RELATED WORK}

Texture Mapping Some techniques have been developed for an automatic mapping of textures onto the model surface. An automatic planar parametrization for surfaces with disk topology based on unfolding the polygon mesh has been concerned in some works 


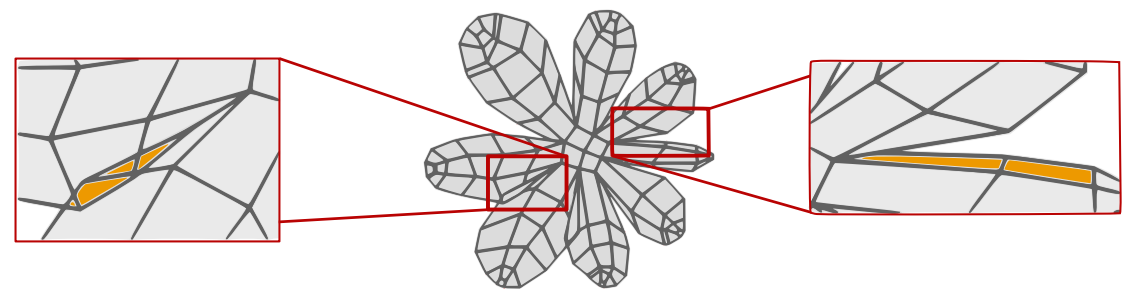

Fig. 2. An automatic atlasing technique fails - polygons either overlap (left) or are distorted (right) if the branching topology in skeleton joint is too complex.

[Sheffer and Sturler 2000], [Lévy et al. 2002], [Floater 2003], while minimizing texture distortion [Lévy and Mallet 1998], stretching and deviation [Sander et al. 2001], defined energy [Maillot et al. 1993] or trying to make the parametrization as isometric as possible [Hormann and Greiner 2000]. Moreover, a sphere is natural domain for genus-0 surfaces, therefore in [Haker et al. 2000] and [Praun and Hoppe 2003] were proposed methods for global spherical parameterization.

When mapping textures onto the surface with an arbitrary topology, two-part texture mapping [Bier and Sloan 1986] can be used, but the method has serious mapping and texture distortion problems with some complex surfaces. In [Ray et al. 2006] authors proposed an automatic global parameterization based on extracting a periodic piecewise linear function. In [Gu and Yau 2003] was solved the problem of computing global conformal parameterizations for surfaces with nontrivial topologies. In addition, the problem of global parametrization was solved in [Jin et al. 2008] using discrete Ricci flow and in [Kharevych et al. 2006] by arranging circles on the surface, one for each face with prescribed intersection angles. Parametrization methods base on relation between curvature and metric was concerned in [Ben-Chen et al. 2008] and [Sheffer and Hart 2002] and a parametrization with focus on minimization of signal approximation error was used in [Sander et al. 2002].

In addition, an arbitrary topology can be parametrized by a piecewise linear domains. Such a domain can be for example a base mesh obtained by mesh decimation [Lee et al. 1998]. However, a mapping of an arbitrary surface onto a plane results into seams. Some works try to solve the seam problem by boundary color duplication [Purnomo et al. 2004], [Carr and Hart 2002] and [Piponi and Borshukov 2000]. Another group of approaches use an octree structure [Benson and Davis 2002], [Debry et al. 2002] and [Lefebvre and Dachsbacher 2007] to store the data instead of mapping the surface into a planar domain. An alternative way to texture mapping was presented in [Yuksel et al. 2010], where the color sample positions are defined directly on the 3D surface. However, such a representation is not suitable when local neighborhoods for each texel are necessary (e.g. filtering, normal estimation from displacement maps), because the local neighborhoods or transition functions between them are not well defined.

The most similar approaches to our skeleton-based texture mapping are [Zhang et al. 2005] and [Tarini et al. 2004]. Zhang et. al [Zhang et al. 2005] used a Reeb graph extracted from mesh features to broke the surface into a set of segments. However, it is not guaranteed that the extracted set of features and the resulting graph correspond with the topology of the model. Similar graph-based parameterization was used by Patane et. el [Patane et al. 
2004], where the Reeb graph was used for the skeletonization of the 2-manifold input mesh and the segments were then parameterized separately. Tirani et. al [Tarini et al. 2004] used cubes around the model to map the texture. A limitation of the polycubes is that they got fixed level of detail over the surface. Another, more limiting drawback is that they encode only the surface, not the topology, therefore they are not general enough to encode topology of an arbitrary model. The shape is composed of axis-aligned unit cubes that are attached face to face. Such a configuration is limiting to branching of model topology, because each cube may have maximum of six neighbors. An automatic construction of polycube maps was introduced in [He et al. 2009] using a divide and conquer strategy. Wang et. al [Wang et al. 2007] made the construction of polycube to be independent of actual geometry of 3D shape allowing different complexity and resolution for the polycube. Lin et. al [Lin et al. 2008] constructed polycube map by segmenting the mesh into a set of patches and then approximating these patches by basic polycube primitives.

A general goal in texture mapping is to get balance between the seams and texture distortion along with solving the problems with triangle overlapping. If the texture mapping domain is subdivided into too much components, the local neighborhoods of texels do not exist and problems with filtering, estimation from displacement map and generating of procedural patterns may occur, because definition of the transaction function may be too difficult. If the number of components is to small, mapping distortion and triangle overlapping problems come into the view. Skeleton segmentation is a natural topology-driven way of cutting a model into a set of components, thus overcoming the above problems.

Skeleton Extraction Numbers of algorithms have been proposed to compute a skeleton from an input mesh geometry. In [Shapira et al. 2008] authors proposed skeleton extraction based on a shape diameter function (SDF). The SDF is a scalar function defined on the mesh surface that expresses a measure of the diameter of the object's volume in the neighborhood of each point on the surface. Thus, a set of random vertices is pushed in an inward normal direction into the volume of the model by a distance that equals to half of the SDF and a least-squares method is used to fit a high-degree curve into the shifted points. Similar approach was used in [Liu et al. 2003], where authors used so called repulsive force field. Sharf et. al [Sharf et al. 2007] introduced a method that is able to perform skeleton extraction on both, point clouds and polygonal meshes. The method uses evolution of a deformable model inside of the mesh. The initial extracted graph is noisy, and extraction of final skeleton require further filtering and merging.

Reeb graph based methods need a suitable real-value function, defined on the model surface for a successful extraction of a skeleton. Using this function, nodes of a $1 \mathrm{D}$ graph can be computed. In [Hilaga et al. 2001] a geodesic function was used for Reeb graph extraction. Alternatively, a method based on a harmonic function proposed by Aujay et. al [Aujay et al. 2007] captures after resampling all the features of the model well, but requires the user to specify the boundary condition explicitly.

$\mathrm{Au}$ et. al [Au et al. 2008] introduced a Laplacian smoothing based method that works directly on the mesh geometry. The main idea of this approach is to apply a well defined filter on mesh vertices. In the first step, an input mesh is contracted using iterative Laplacian contraction. Then, a mesh decimation is used to simplify the contracted mesh into a curve-skeleton. Cao et. al [Cao et al. 2010] extended the idea of Laplacian contraction 
[Au et al. 2008] for a point cloud input. They used a definition of the Laplacian operator for a point cloud in order to perform a similar weighted filtering. When the mesh is contracted, mesh decimation cannot be used, because there is not defined an edge connectivity. Authors made selection of contracted points to be connected based on their euclidean distance. Therefore, the ability to extract a proper skeleton depends on the distance between samples on the manifold being smaller than the distance between the two structures.

In [Teichmann and Teller 1998] authors extract skeleton by simplifying the Voronoi skeleton with a small amount of user assistance. Another Voronoi diagram based method [Dey and Sun 2006] compute the skeleton from the medial axis obtained by extracting the internal edges of the Voronoi diagram. These methods are quite slow in comparison to Reeb graph or Laplacian smoothing based ones and do not guarantee that the result will capture all desired features.

\section{SKELETON TEXTURE MAPPING}

Skeleton texture mapping works as follows. First, a skeleton is extracted from an input mesh (Section 3.1). The skeleton is used for a segmentation of the model in a topologydriven way and to define the axis of each segment. A rectangular area in the final texture is reserved for each segment by solving a packing problem (Section 3.2). Then, geometry around each skeleton segment is mapped into a rectangular texture using capsule parameterization (Section 3.3). A global STM coordinates are computed by shifting each rectangle from local rectangle coordinates into the positions precomputed by segment packing algorithm. During the mapping, topological constraints resulting into seams are resolved by doubled rendering of triangles that lie on the seams (Section 3.4). Finally, local neighborhoods of each texel is guaranteed by mirroring and boundary color duplication (Section 3.5). In the end, a discussion on robustness of our method can be found (Section 3.6).

\subsection{Skeleton Extraction}

For our method the Laplacian smoothing based extraction is the best choice, because the skeleton nodes are created by shrinking the original mesh vertices into the models volume and merging into skeleton nodes. Therefore, these methods give us the one-to-many mapping between skeleton segments and mesh vertices for free. Using some other method, it would require to find the association after the skeleton extraction.

That is why we have chosen [Au et al. 2008] as the base of the skeleton extraction algorithm. The algorithm runs in two steps. In the first step, the input mesh is contracted using an iterative Laplacian smoothing in few iterations. In each iteration, a Laplacian operator is constructed for each vertex and applied on the mesh. When the iteration process converges, the contracted mesh is homotopic to the original mesh and has almost zero volume. In the second step, the skeleton is constructed by simplifying the contracted mesh using a skeleton decimation algorithm [Garland and Heckbert 1997] with one change. The error matrices are computed over the edges, because the contracted mesh has zero area faces, so the original volume based algorithm cannot be used.

In addition, in the skeleton construction stage, we have to guarantee that each skeleton segment lies inside the mesh volume. Every iteration of the greedy algorithm, which is 
used for mesh simplification, we check if merging the vertex into another one does not move the skeleton segment out of the volume. If such a situation is detected, we forbid the merging of these vertices and the greedy algorithm jump to next pair of vertices with the lowest cost estimation.

\subsection{Packing Problem}

Given the skeleton segments and geometry associated with the segments, we map each segment triangles into a rectangular texture and pack all these rectangles into one final square texture. A rectangular texture with higher priority needs better storing of details, hence it will be stored in a bigger area than rectangle with lower priority. Determining relative size ratio between the rectangles, we can formulate the storing of these rectangles as a 2-dimensional distributor's pallet loading problem [Birgin et al. 2010] of storing $N$ rectangles with size $\left(R_{i}^{W}, R_{i}^{H}\right)$ into a unit square. Thus we maximize the sum of the area they cover (Equation 1) and we can scale them by a global constant $s \in R^{+}$

$$
\underset{s}{\arg \max } \sum_{i=0}^{N-1} s^{2} R_{i}^{W} R_{i}^{H} \leq 1
$$

We use a binary search to find the correct scaling ratio $s$ to fit the rectangles. The placing of the rectangles is done using a k-D tree and a recursive fitting (Figure 3). We explore the configurations for assigned $s$ and if there is an acceptable configuration, we increase $s$ and iterate the fitting again. If there is no next acceptable configuration, we take the last one as the solution.

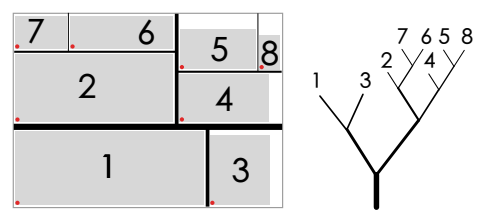

(a) (b)

Fig. 3. Remapped rectangular textures are packed into the squared texture (a) using a k-D tree (b).

\subsection{Segment Mapping}

A capsule around each skeleton segment is mapped into a rectangle, the central part uses cylindrical coordinates and two ends use the mapping of spherical caps as shown in Figure 4. Furthermore, the relative texture coordinates inside each rectangle are computed as in Equation 2 and Equation 3: 


$$
\begin{aligned}
u & =\frac{\varphi}{2 \pi} h \\
v & =\left\{\begin{array}{ll}
d\left(1-\frac{\theta_{1}}{\pi / 2}\right) & t<0 \\
d+t(w-2 d) & t \in<0,1> \\
w+d\left(\frac{\theta_{1}}{\pi / 2}-1\right) & t>1 .
\end{array}\right\},
\end{aligned}
$$

where $w$ and $h$ determine the size of the rectangle and $d$ influences the area that is reserved for the part of the texture that is encoding the capsule caps. Cylindrical coordinates describing the central part are $(\varphi, t)$ and spherical coordinates describing cups are $\left(\varphi, \theta_{1}\right)$.

For each skeleton segment priority weights are computed from the surface area ratio and the segment length, which are used to determine the size of the rectangle and the cap. Furthermore, when storing texture of the segment caps, the cap triangles must have at least one vertex that does not lie on a circle with other two vertices. Such a triangle has all three angles $\theta=0$ and is rendered into STM as a line. To overcome this problem, such a special case can be detected and additional rectangle with different transformation can be used to encode these cap triangles. For example the same spherical projection with rotated bases or an orthogonal projection can be used.

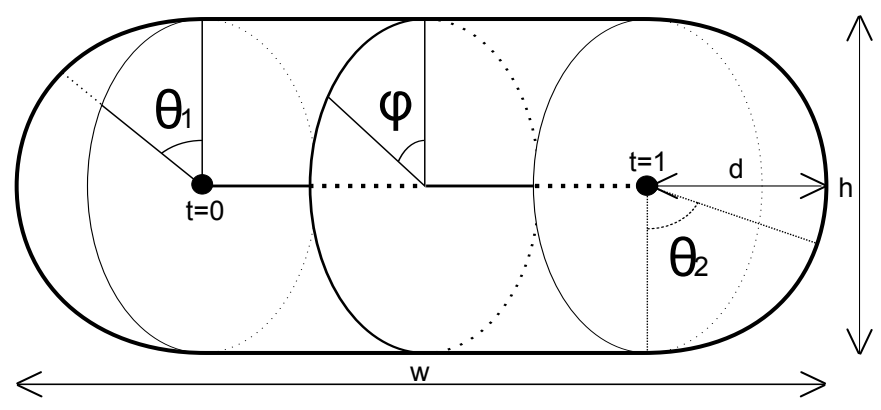

Fig. 4. Capsule parametrization along a skeleton segment between two nodes. Texture coordinates are computed from the rectangle and cap size and the pairs of angles $\theta_{i}, \varphi$.

\subsection{Topology Constraints}

In order to make the mapping as effective as possible, we use the rasterizer to interpolate the values in the texture. Moreover, during the mapping of the capsule surface around the skeleton onto a planar texture, seams are inevitable. Triangles that lie on texture seam can not be rendered in a normal way, because it's $u v$ coordinates lie on the opposite sides of texture and rasterizer interpolates texels through whole texture. It is not possible to tell the rasterizer to interpolate texels in the opposite way, through the seam. Thus, we propose three ways how to solve this problem. First solution concerns generating two textures for each segment (Figure 5(a)), where the first seam is cut at angle $\varphi=0$ and the second one at $\varphi=\pi$. The biggest possible angle difference between two vertices in one triangle is $\pi$ and therefore if the seam occurs in one texture, it will not occur in the other one. The second solution uses the cube maps to encode texture around the skeleton (Figure 5(b)). In 
this case the seam the problem is solved by hard-wired hardware algorithm. We choose a third solution, because it was the easiest to implement and the most efficient one. We split the texture at $\varphi=0$ and all mesh triangles that lie on the seam has to be treated in a special way. If an input mesh shares vertices through indices, vertices of seam triangles on one side of the seam have to be duplicated (Figure 6(a)) and different texture coordinates are assigned to the duplicated vertices to cover both sides of the texture seam. Triangle will be also duplicated during rendering into STM to cover both sides of the texture (Figure 6(b)).

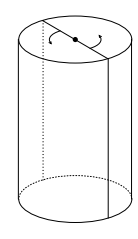

(a)

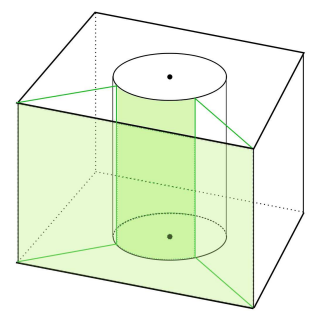

(b)

Fig. 5. Each segment can be encoded into two textures (a) or hardware cube mapping can be used (b).

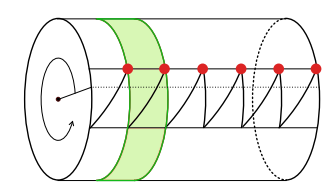

(a)

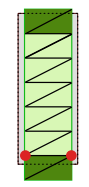

(b)

Fig. 6. Surface with cylindrical topology has to be cut (a). Triangles that lie on the resulting seam (b - dark green) have to be duplicated. During the rendering the vertices on the right side of the seam (red dots) are send to GPU twice with different $u v$ coordinates.

\subsection{Segment Composing and Mirroring}

Initially we render each skeleton segment into its own rectangular texture. Knowing the area where each rectangle should lie (calculated in Section 3.2), the rectangle is rendered into this area. We set a global mirroring parameter, which determines the percentage, how much of the rectangle should be used for mirroring area. Thus, we clip each rectangle area by this mirroring margin before rendering and render the rectangle into the clipped area only. Furthermore, we perform topologically correct mirroring in the margin area. Part of the texture that topologically belongs here is rendered into each mirroring margins. This means that in horizontal direction the texture is enclosed by mirrored parts of itself and in the vertical direction by texture parts of the neighboring segments. Performing this mirroring we achieve that each texel in the final texture is surrounded by texels that really lie in the closest neighborhood of the point on the model surface.

In the case of skeleton texture mapping, the transition function is defined by deterministic allocation of rectangles in the final texture. The jumping from one chart to the neighboring one can be done by searching for the neighbors in the skeleton structure. The skeleton 
structure gives us an id of neighboring segment and each segment has its own fixed coordinates in the texture space.

\subsection{Discussion on Robustness}

In this section we prove robustness of our approach. Consider an input closed 2D manifold mesh topologically equivalent to a sphere with $N$ handles. If the mesh is disconnected, a skeleton is computed for each component separately and then all the skeletons are packed into the same STM. For each handle a skeleton is constructed, where the first and the last nodes are overlapping and creating a cycle around the handle. The remaining geometry without handles can be always decomposed into cylinders and described by capsule-shaped domain. The robustness can be proved by mathematical induction. First we cover all the handles by segments and then all the remaining parts can be decomposed into cylinders, until we cover the whole model.

However, the whole idea of the algorithm is based on a fact that the extracted skeleton is reliable [Cornea et al. 2007]. Reliability refers to the property of the curve-skeleton that every boundary point (point on objects surface) is visible from at least one curve-skeleton location. In other words, for any boundary point, there exists a straight line connecting it to a curve-skeleton point that does not cross any boundary. The second property of the skeleton is the centeredness. The skeleton does not have to be centred perfectly, but more centered skeleton yields less distorted mapping. However, the skeleton has to lie within the model. This is guaranteed by shifting of skeleton nodes into the center of mass of vertices, they were merged of. Furthermore, during the skeleton simplification we check, if the simplification does not cause penetration of the surface by the skeleton segment. If such a situation happens, the current step of simplification is skipped.

The global robustness of our approach may be violated by two facts. The first fact is that currently the skeleton can be correctly extracted from 2-manifold meshes only. This can be improved by a modification of the skeleton extraction algorithm to support a skeleton extraction from point clouds. The second and more relevant fact is that the extracted curveskeleton may lay out of the model in some special cases. The skeleton contraction process may produce a skeleton lying outside of the volume for certain objects where some local regions have the center-of-mass outside the object (e.g., one with a C-shape cross-section). Even the shifting of skeleton nodes into the center of mass cannot help, because the center of mass of the C-shape local cross section lies outside of the model. However, the skeleton extraction centers the skeleton well for most organic-shaped models [Au et al. 2008], where the texture mapping is relevant.

\section{RESULTS}

Here we present an applications of skeleton texture mapping concerning remapping of textures. Given an input model that contains texture and stored $u v$ coordinates for each vertex we extract a skeleton and STM. Later, we can remove $u v$ coordinates from the data structure representing the model and the model can be rendered using STM. When rendering, the skeleton will be computed in the preprocessing stage and new STM $u v$ coordinated can be computed during rendering. 

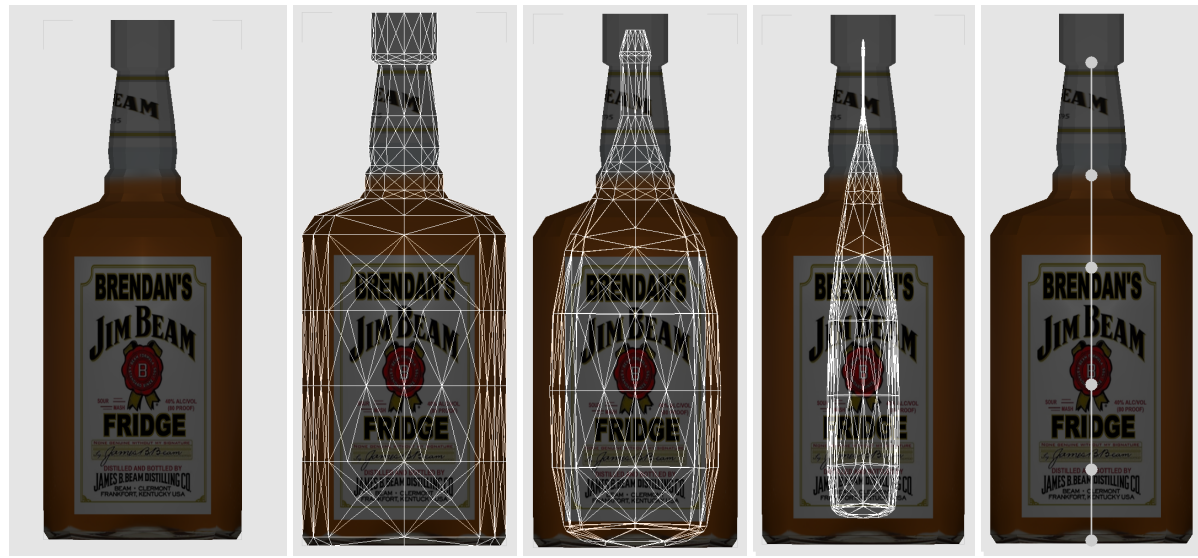

Fig. 7. From left to right: an original textured model, few steps of the skeletonization process, the final skeleton.
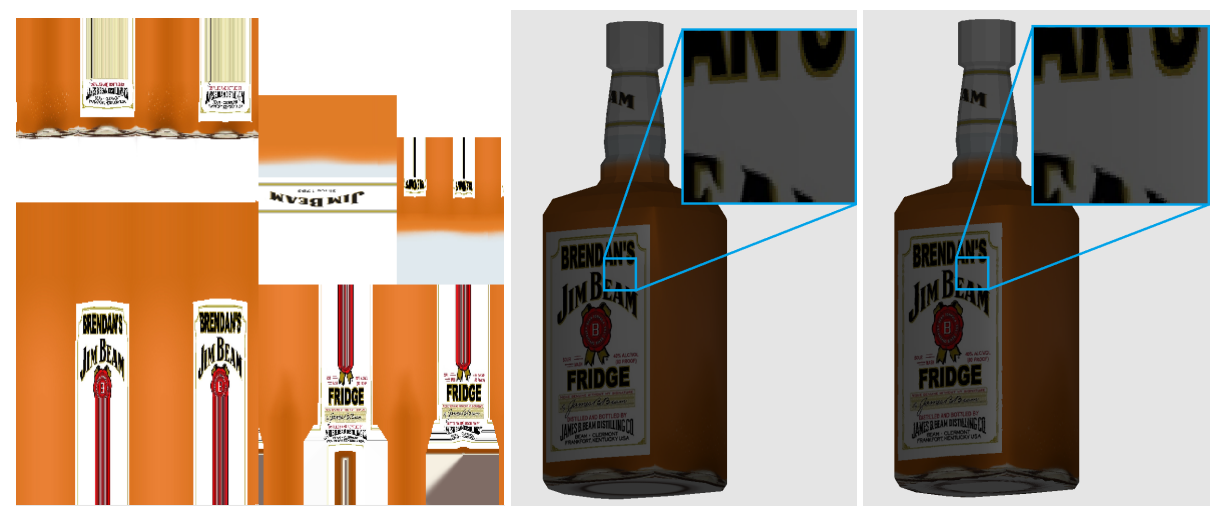

Fig. 8. From left to right: extracted STM, model textured using original texture and uv coordinates with zoomed area, model textured using our STM approach with zoomed area.

First, we compute STM coordinates using our proposed technique. Second, the model is rendered into a fragment buffer object (FBO). As vertex positions the computed STM coordinates are used. The rasterization interpolates the texture values over the STM. During the STM mapping back to model, vertices near skeleton nodes can be mapped to more segment rectangles. We consider the one with the best mapping properties, where the texture is remapped with the lowest distortion. It is a segment where dot product between the skeleton segment vector and the triangle normal is minimal. The whole skeletonization process can be seen in Figure 7. An extracted STM and comparison of the model textured with original texture and remapped texture onto the model surface using proposed STM can be seen in Figure 8. Currently implemented algorithm for skeleton extraction works only with 2-manifold meshes and the iterative contraction is very time expensive operation for high resolution models. Therefore, we show results only on simple primitives with variety of extreme geometric properties (Figure 9). 

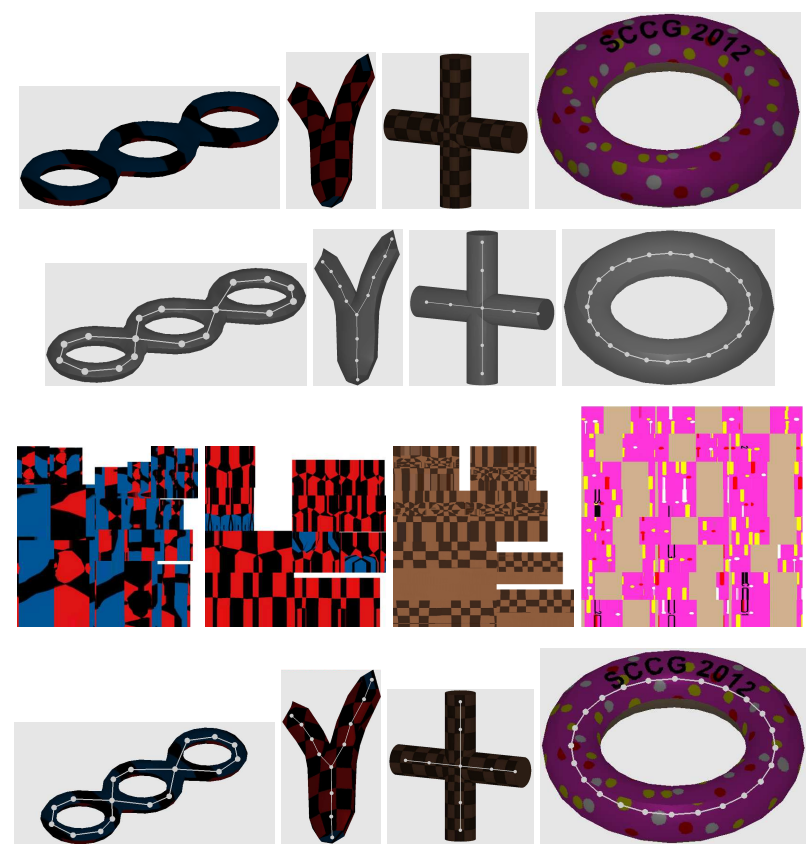

Fig. 9. Here we show results of our method on some primitives. In rows from top to bottom: an input model, extracted skeleton, remapped STM, model textured using skeleton texture mapping.

\subsection{Computation Time}

Table 1 shows how much time each stage of skeleton texture mapping requires. The time was measured on an AMD Phenom II X4 3.41GHz with 4GB RAM, using a single thread implementation for the calculations. As we can see, the most time consuming parts of the implementation is solving the linear system (contraction of the mesh).

\begin{tabular}{|l|r|r|r|r|r|r|}
\hline Model & \#vert. & $t_{1}$ & $t_{2}$ & $t_{3}$ & $t_{4}$ & Total \\
\hline \hline Ypsilon & 480 & $<0.1$ & $<0.1$ & 0.2 & 2.1 & 2.6 \\
JB bottle & 1530 & 2.2 & 0.9 & 0.6 & 2.8 & 6.4 \\
Donut & 1728 & 8.4 & 1.6 & 0.4 & 2.1 & 12.6 \\
Triple-torus & 2256 & 16.5 & 4.8 & 0.3 & 2.4 & 24.1 \\
Crossed cyl. & 3660 & 73.5 & 7.0 & 0.5 & 3.6 & 84.7 \\
\hline
\end{tabular}

Table I. Columns $t_{1}, t_{2}, t_{3}, t_{4}$ and $t_{5}$ show the running time (in seconds), mesh contraction, skeleton construction, solving the packing problem and skeleton texture composing respectively.

\subsection{Packing Efficiency}

Our algorithm does not solve the packing problem ideally, but for a set of testing models texture coverage varies from $75 \%$ to $99 \%$. Such an efficiency is sufficient for our applications of STM and for any further work with it. Measured packing efficiency in comparison to number of segments can be seen in Table 2. 


\begin{tabular}{|l|r|r|}
\hline Model & \# segments & \% of area coverd in STM \\
\hline \hline Donut & 24 & $99.7 \%$ \\
Triple-torus & 24 & $91 \%$ \\
JB Bottle & 5 & $89.4 \%$ \\
Ypsilon & 7 & $83.7 \%$ \\
Crossed cylinders & 12 & $74.3 \%$ \\
\hline
\end{tabular}

Table II. Table shows the packing efficiency of our algorithm for solving the packing of $N$ rectangles into one squared texture. It is easier to pack many smaller segments than few bigger ones.

\section{APPLICATIONS AND FUTURE WORK}

There are many useful applications of STM in computer graphics. The mapping can be used for extracting a STM from a textured mesh with classical $u v$ coordinates and applying the STM onto a surface without parametrization. Furthermore capsule-shaped domain around each segment can be used for procedural generating of textures aligned by skeleton axis. Using our technique, such a procedurally generated texture can be mapped around arbitrary skeleton segment. The model geometry is not necessary while generating the texture.

Another group of applications concern skeleton displacement map (SDM), a case where texture encodes vertex displacement from skeleton. SDM can be used as a data structure for model representation. The skeleton and SDM are extracted from an input model and later they are used for reconstruction of the original model surface. Thus, a per segment level of detail can be assigned to the skeleton during reconstruction stage.

Notice that such an applications are neither possible with another global parameterization methods nor Reeb graph based parameterization [Patane et al. 2004]. To our knowledge, the polycube maps is the only technique with such possible applications, however with constrained topology with maximum of six skeleton branches per node.

As the future work we would like to explore another mappings for the segment caps and additionally implement encoding of the caps based on cube and sphere maps. In addition, during the capsule mapping we would like to explore benefits of a least-squares conformal mapping [Lévy et al. 2002] and compare the performance and quality of the results of these approaches.

Furthermore, we plan to implement a general skeleton extraction algorithm based on skeleton extraction algorithm from point clouds [Cao et al. 2010]. Such an implementation would be able to extract correct skeletons also from non-manifold meshes. In addition, we would like to parallelize whole skeleton extraction process using OpenCL. By improving this two major drawbacks of the algorithm, we would get fast and robust algorithm for skeleton extraction from a general input geometry as a non-manifold mesh, a polygon soup or a point cloud. Thus, we would be able to perform the skeleton texture mapping on variety of more complex and challenging models in order to make a comparison with another texture mapping methods and further evaluation of mapping distortion. 


\section{ACKNOWLEDGMENT}

Our project was partly supported by project VEGA 1/0438/13 a Scientific grant from Ministry of Education of Slovak Republic and Slovak Academy of Science and from the grant Comenius University grant UK/121/2012.

\section{REFERENCES}

AU, O. K.-C., TAI, C.-L., ChU, H.-K., COHEN-OR, D., AND LEE, T.-Y. 2008. Skeleton extraction by mesh contraction. In ACM SIGGRAPH 2008 papers. 1-10.

Aujay, G., Hétroy, F., Lazarus, F., and Depraz, C. 2007. Harmonic skeleton for realistic character animation. In Proceedings of the 2007 ACM SIGGRAPH. 151-160.

Ben-Chen, M., Gotsman, C., And Bunin, G. 2008. Conformal flattening by curvature prescription and metric scaling. Computer Graphics Forum 27, 2, 449-458.

Benson, D. And Davis, J. 2002. Octree textures. ACM Trans. Graph. 21, 785-790.

BIER, E. AND SLOAN, K. 1986. Two-part texture mappings. IEEE Computer Graphics and Applications 6, $40-53$.

Birgin, E. G., Lobato, R. D., AND Morabito, R. 2010. Generating unconstrained two-dimensional nonguillotine cutting patterns by a recursive partitioning algorithm.

CaO, J., Tagliasacchi, A., Olson, M., Zhang, H., And Su, Z. 2010. Point cloud skeletons via laplacian based contraction. In Proceedings of the 2010 SMI Conf. 187-197.

CARR, N. A. AND HART, J. C. 2002. Meshed atlases for real-time procedural solid texturing. ACM Transactions on Graphics 21, 106-131.

Cornea, N. D., Silver, D., And Min, P. 2007. Curve-skeleton properties, applications, and algorithms. IEEE Transactions on Visualization and Computer Graphics 13, 3, 530-548.

Debry, D., Gibbs, J., Deleon, D., And Robins, P. N. 2002. Painting and rendering textures on unparameterized models.

DEY, T. K. AND SUn, J. 2006. Defining and computing curve-skeletons with medial geodesic function. In Proceedings of the 4th EG symposium on Geom. processing. 143-152.

Floater, M. S. 2003. Mean value coordinates. Computer Aided Geometric Design 20, 2003.

GaRland, M. AND HeckBerT, P. S. 1997. Surface simplification using quadric error metrics. In SIGGRAPH '97: Proceedings of the 24th annual conference on Computer graphics and interactive techniques. ACM Press/Addison-Wesley Publishing Co., New York, NY, USA, 209-216.

Gu, X. And YaU, S.-T. 2003. Global conformal surface parameterization. In Proceedings of the 2003 Eurographics/ACM SIGGRAPH symposium on Geometry processing. SGP '03. Eurographics Association, Aire-laVille, Switzerland, Switzerland, 127-137.

Haker, S., Angenent, S., Tannenbaum, A., Kikinis, R., Sapiro, G., and Halle, M. 2000. Conformal surface parameterization for texture mapping. IEEE Transactions on Visualization and Computer Graphics 6 , $181-189$.

HE, Y., WANG, H., FU, C.-W., AND QIN, H. 2009. A divide-and-conquer approach for automatic polycube map construction. Computers \& Graphics, 369-380.

Hilaga, M., Shinagawa, Y., Kohmura, T., And Kunit, T. L. 2001. Topology matching for fully automatic similarity estimation of $3 \mathrm{~d}$ shapes. In Proceedings of the 28th annual conference on Computer graphics and interactive techniques. SIGGRAPH '01. ACM, New York, NY, USA, 203-212.

Hormann, K. And Greiner, G. 2000. MIPS: An Efficient Global Parametrization Method. Vanderbilt University Press.

Jin, M., KIM, J., LUO, F., AND GU, X. 2008. Discrete surface ricci flow. IEEE Transactions on Visualization and Computer Graphics 14, 1030-1043.

Kharevych, L., Springborn, B., AND Schröder, P. 2006. Discrete conformal mappings via circle patterns. ACM Trans. Graph. 25, 412-438.

LeE, A. W. F., SWeldens, W., Schröder, P., Cowsar, L., ANd Dobkin, D. 1998. MAPS: multiresolution adaptive parameterization of surfaces. In Proceedings of the 25th annual conference on Computer graphics and interactive techniques. SIGGRAPH '98. ACM, New York, NY, USA, 95-104. 
Lefebvre, S. And Dachsbacher, C. 2007. Tiletrees. In Proceedings of the ACM SIGGRAPH Symposium on Interactive $3 D$ Graphics and Games.

LÉVy, B. AND MALlET, J.-L. 1998. Non-distorted texture mapping for sheared triangulated meshes. In Proceedings of the 25th annual conf. on CG and interactive techniques. 343-352.

LÉvy, B., Petitjean, S., Ray, N., And Maillot, J. 2002. Least squares conformal maps for automatic texture atlas generation. ACM Trans. Graph. 21, 362-371.

LIN, J., Jin, X., FAN, Z., AND WANG, C. C. L. 2008. Automatic polycube-maps. In Proceedings of the 5th international conference on Advances in geometric modeling and processing. GMP'08. Springer-Verlag, Berlin, Heidelberg, 3-16.

LiU, P.-C., Wu, F.-C., MA, W.-C., LiAng, R.-H., AND Ouhyoung, M. 2003. Automatic animation skeleton construction using repulsive force field. In Proceedings of the 11th Pacific Conference on CG and Applications. 409-413.

Maillot, J., Yahia, H., And Verroust, A. 1993. Interactive texture mapping. In Proceedings of the 20th annual conference on Computer graphics and interactive techniques. 27-34.

Patane, G., Spagnuolo, M., And Falcidieno, B. 2004. Para-graph: Graph-based parameterization of triangle meshes with arbitrary genus. Comput. Graph. Forum, 783-797.

PiPONI, D. AND Borshukov, G. 2000. Seamless texture mapping of subdivision surfaces by model pelting and texture blending. In Proceedings of the 27th annual conference on Computer graphics and interactive techniques. SIGGRAPH '00. ACM Press/Addison-Wesley Publishing Co., New York, NY, USA, 471-478.

Praun, E. AND Hoppe, H. 2003. Spherical parametrization and remeshing. ACM Trans. Graph. 22, 340-349.

Purnomo, B., Cohen, J. D., AND KumAR, S. 2004. Seamless texture atlases. In ACM SIGGRAPH symposium on Geometry processing. 65-74.

Ray, N., Li, W. C., LÉvy, B., Sheffer, A., AND Alliez, P. 2006. Periodic global parameterization. ACM Trans. Graph. 25, 1460-1485.

Sander, P. V., Gortler, S. J., Snyder, J., And Hoppe, H. 2002. Signal-specialized parametrization. In Proceedings of the 13th Eurographics workshop on Rendering. EGRW '02. Eurographics Association, Airela-Ville, Switzerland, Switzerland, 87-98.

Sander, P. V., SNyder, J., Gortler, S. J., AND Hoppe, H. 2001. Texture mapping progressive meshes. In Proceedings of the 28th annual conf. on CG and inter. techn. 409-416.

Shapira, L., Shamir, A., And Cohen-Or, D. 2008. Consistent mesh partitioning and skeletonisation using the shape diameter function. Vis. Comput. 24, 249-259.

Sharf, A., Lewiner, T., Shamir, A., And Kobbelt, L. 2007. On-the-fly curve-skeleton computation for 3D shapes. Computer Graphics Forum, (Proceedings Eurographics 2007) 26, 3, 323-328.

ShEFFER, A. AND HART, J. C. 2002. Seamster: Inconspicuous low-distortion texture seam layout. In IEEE Visualization. 291-298.

SHEFFER, A. AND STURLER, E. D. 2000. Surface parameterization for meshing by triangulation flattening. In Proc. 9th International Meshing Roundtable. 161-172.

Tarini, M., Hormann, K., Cignoni, P., And Montani, C. 2004. Polycube-maps. In In Proceedings of SIGGRAPH 2004. 853-860.

TEIChmann, M. AND TELler, S. 1998. Assisted articulation of closed polygonal models. In SIGGRAPH '98: ACM SIGGRAPH 98 Conference abstracts and applications. ACM, New York, NY, USA, 254.

Wang, H., He, Y., LI, X., Gu, X., AND QIN, H. 2007. Polycube splines. In Proceedings of the 2007 ACM symposium on Solid and physical modeling. SPM '07. ACM, New York, NY, USA, 241-251.

Yuksel, C., Keyser, J., AND House, D. H. 2010. Mesh colors. ACM Trans. Graph. 29, 15:1-15:11.

ZhANG, E., Mischaikow, K., AND TURK, G. 2005. Feature-based surface parameterization and texture mapping. ACM Trans. Graph. 24, 1-27.

Authors' addresses:

Martin Madaras

Mathematics, Physics and Informatics,

Comenius University,

84248 Bratislava, Slovak Republic 
http://www.fmph.uniba.sk

email:madaras@sccg.sk

Roman Ďurikovič

Faculty of Mathematics, Physics and Informatics,

Comenius University,

84248 Bratislava, Slovak Republic

http://www.fmph.uniba.sk

email: roman.durikovic@fmph.uniba.sk 